\title{
étude théorique et en laboratoire du comportement d'une fracture rocheuse sous contrainte normale
}

\author{
D. BILLAUX - B. FEUGA \\ BRGM - Orléans \\ S. GENTIER \\ BRGM et Université d'Orléans
}

\section{INTRODUCTION}

Un certain nombre de grands projets de recherche actuellement en cours amènent à s'intéresser au comportement sous contrainte normale des fractures dans les milieux rocheux.

C'est le cas en particulier des études liées aux projets de stockage des déchets radioactifs dans le sous-sol ou à ceux visant l'exploitation de la chaleur contenue dans les couches profondes de l'écorce terrestre. Dans ces projets, en effet, des variations de contrainte, dues par exemple à des modifications de température ou de pression interstitielle, peuvent modifier l'ouverture des fractures et entrainer de ce fait des changements dans la perméabilité des terrains qụi peuvent être de première importance pour les projets en question. Ces variations d'ouverture peuvent être liées à des cisaillements (dilatance-contractance), mais également à des modifications des seules contraintes normales. C'est cet aspect du problème qui est abordé dans cet article. Le programme de recherche dont les premiers résultats sont présentés ici a pour objet de mettre au point un modèle de comportement d'une fracture en compression, fondé sur la morphologie de cette fracture et les caractéristiques mécaniques du matériau rocheux, qui puisse être introduit dans des simulations de milieux rocheux fracturés.
Le matériau testé est un granite à grains fins du Limousin. On présente les résultats des déterminations de profils relevés sur une fracture naturelle dans ce granite, les essais de compression simple réalisés sur cette fracture, et enfin deux modèles dont l'un semble reproduire convenablement les résultats de ces essais.

\section{DESCRIPTION D'UNE FRACTURE}

On assimile une fracture à deux épontes planes indéformables dotées de dents de hauteur $\mathrm{h}$ variable (fig. 1). Les deux épontes sont situées à une distance $e$ l'une de l'autre; cette grandeur $e$, qu'on appellera "écartement" de la fracture est donc égale à la hauteur du plus grand des vides. Elle dépend évidemment de la contrainte normale $\sigma_{\mathrm{N}}$ appliquée aux épontes. On appelle d la hauteur du vide laissé entre les épontes par une dent de hauteur h (fig. 1). On appelle $e_{0}$ l'écartement maximal, qui n'est autre que la valeur de e sous contrainte nulle.

On norme les grandeurs $e, d$ et $h$ par $e_{0}$. Il vient: $e^{+}=\frac{e}{e_{0}}, \quad d^{+}=\frac{d}{e_{0}}, h^{+}=\frac{h}{e_{0}}$

$\mathrm{e}^{+}, \mathrm{d}^{+}$et $\mathrm{h}^{+}$sont dont compris entre 0 et 1 , et $\mathrm{d}^{+}+\mathrm{h}^{+}=\mathrm{e}^{+}$. 


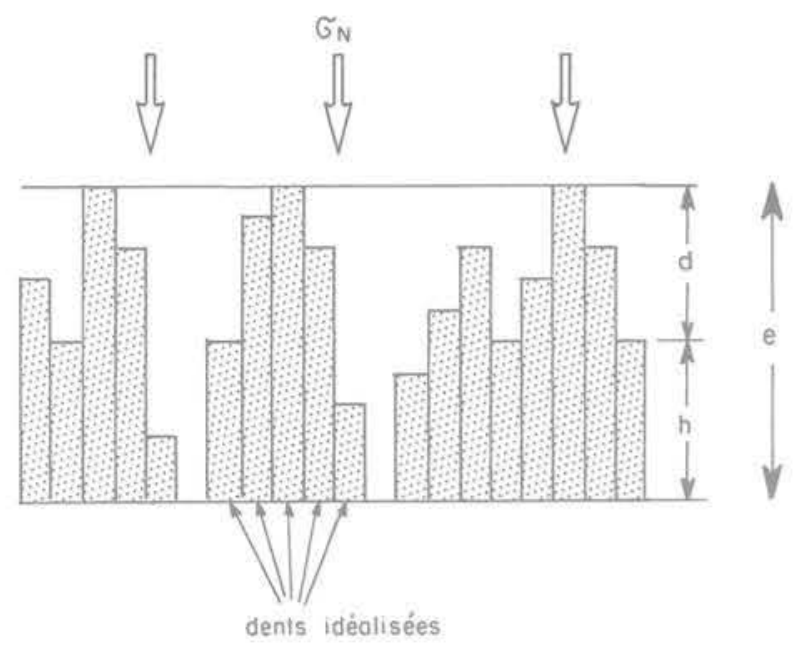

Fig. 1. - Fracture idéalisée.

Les termes "écartement", "hauteur de dent » et "hauteur de vide» s'appliquent, dans la suite de l'exposé, aux grandeurs normées, les hauteurs de dents étant de plus des hauteurs sous contrainte nulle, sauf mention contraire. Pour un écartement donné $\mathrm{e}^{+}$, on appelle degré d'ouverture (ou degré de séparation) $\tau\left(e^{+}\right)$le rapport, dans le plan de la fracture, entre la surface ouverte et la surface totale de la fracture. $\tau\left(e^{+}\right)$est donc la fraction de la surface sur laquelle les dents ont une hauteur sous contrainte nulle inférieure à $e^{+}$. On peut donc déterminer les variations de $T$ en fonction de $e^{+}$à partir de la distribution des hauteurs de dent, ou de celle des hauteurs de vide.

\section{3. ÉTUDE EN LABORATOIRE DES PROFILS DES FRACTURES ET DE LEUR COMPORTEMENT EN COMPRESSION SIMPLE}

La première série d'essais a porté sur des éprouvettes fracturées de granite à grain moyen $(2$ à $3 \mathrm{~mm}$ ) à tendance porphyroïde provenant de la carrière de Maupuy dans les environs de Guéret (Creuse) et ayant pour caractéristiques moyennes:

$\mathrm{E}=63800 \mathrm{MPa}$ (s = $900 \mathrm{MPa}$ ):

$v=0,22(s=0,02)$

$\sigma_{c}=179 \mathrm{MPa}(\mathrm{s}=13 \mathrm{MPa})$

Ces caractéristiques ont été déterminées à partir d'essais de compression simple effectués sur six éprouvettes intactes $(\varnothing=12 \mathrm{~cm}$ pour $E$ et $v, \varnothing=5 \mathrm{~cm}$ pour $\sigma_{c}$, élancement 2).

Les éprouvettes utilisées pour l'étude des fractures ont toutes été carottées, en diamètre $12 \mathrm{~cm}$, dans un même bloc de granite comportant une fracture naturelle ouverte, l'axe des éprouvettes étant perpendiculaire à la fracture.
Le processus expérimental comprenait l'acquisition de "profils de rugosité » de la fracture afin d'établir une distribution des hauteurs de vide, puis des essais en compression simple permettant d'établir une relation entre contrainte normale et écartement de la fracture, et enfin un nouveau relevé des «profils de rugosité après compression.

3.1. Détermination des "profils de rugosité " et de la distribution des hauteurs de vide $\mathrm{g}\left(\mathrm{d}^{+}\right)$

Le arugosimètre v utilisé pour l'enregistrement des profils est constitué d'un palpeur solidaire d'un chariot se déplaçant horizontalement le long d'une vis sans fin. Le contrôle de ce déplacement est assuré par un capteur linéaire d'une précision de l'ordre de $0,4 \mathrm{~mm}$ pour une course totale de $100 \mathrm{~mm}$. Le contrôle du déplacement vertical du palpeur est assuré par un capteur linéaire d'une précision de $0,02 \mathrm{~mm}$ pour une course totale de $6 \mathrm{~mm}$. L'ensemble des déplacements est recueilli sur une table traçante (fig. 2, 3, 4).

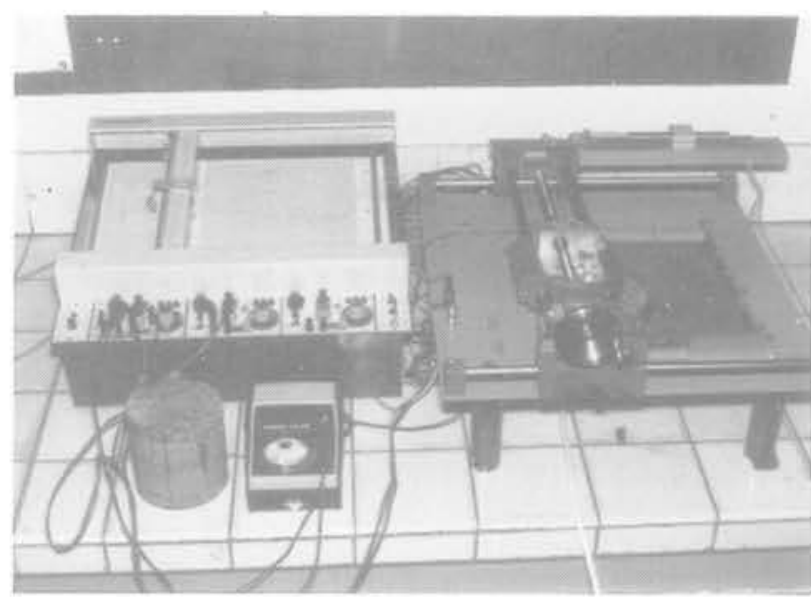

Fig. 2. - Vue d'ensemble du système d'enregistrement des profils (rugosimètre et table traçante).

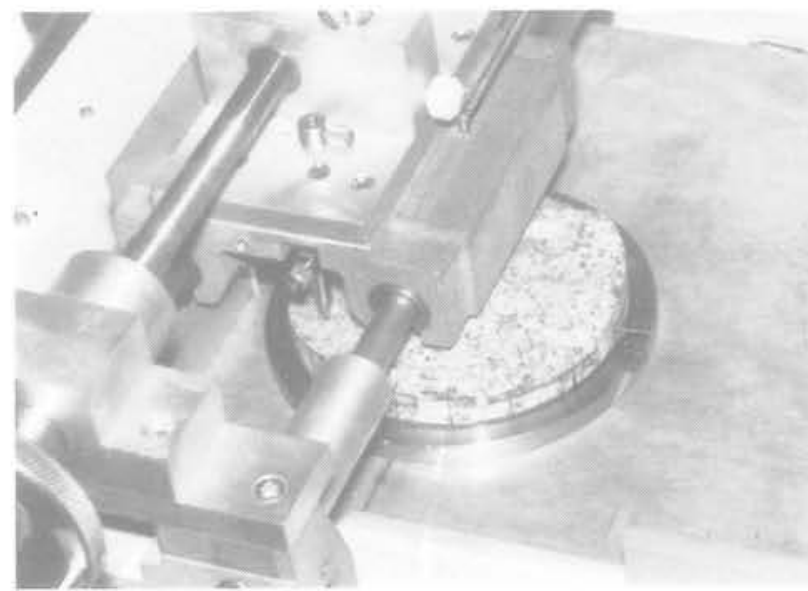

Fig. 3. - Détail du rugosimètre: chariot permettant le déplacement horizontal et palpeur. 


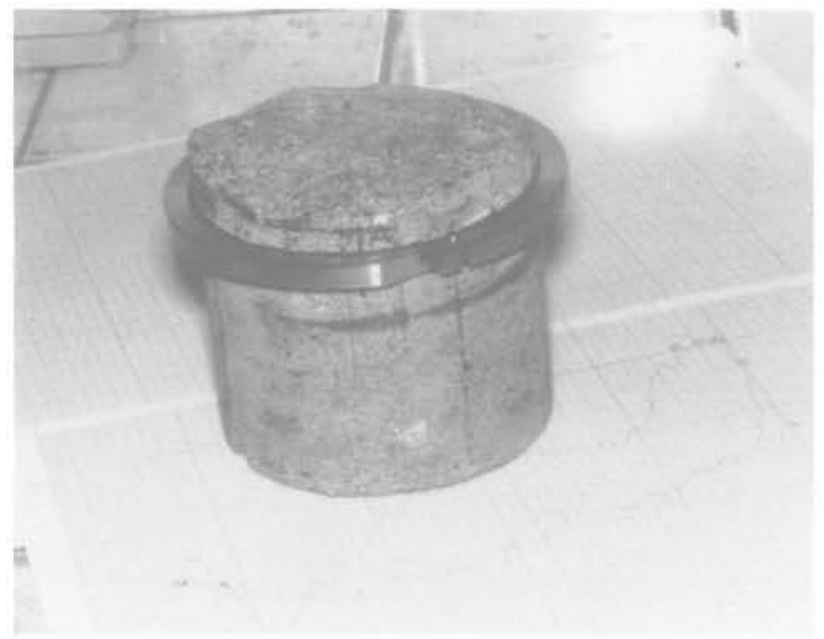

Fig. 4. - Demi-éprouvette munie de son anneau de fixation.
Sur chacune des lèvres de la fracture, on déplace le palpeur suivant deux séries de profils $(A B$ et $C D)$ constituées chacune de cinq profils paralleles entre eux. Les deux séries sont perpendiculaires entre elles dans le plan de la fracture (fig. 5). Le calage des deux ensembles de profils (un ensemble pour chacune des lèvres de la fracture) l'un par rapport à l'autre donne des images de la fracture équivalentes à des sections (fig. 6).

La direction de mesure des hauteurs de vide est parallèle à l'axe de l'éprouvette et correspond à la direction d'application de la contrainte lors des essais mécaniques. Pour chacune des directions d'enregistrement $(A B$ et $C D)$ on établit une distribution moyenne des hauteurs de vide. A partir de ces deux distributions moyennes, on calcule une distribution moyenne globale des hauteurs de vide sous contrainte nulle (fig. 7). Les deux directions d'enregistrement donnent des distributions moyennes de forme générale semblable avec cependant plus de grandes hauteurs de vide pour

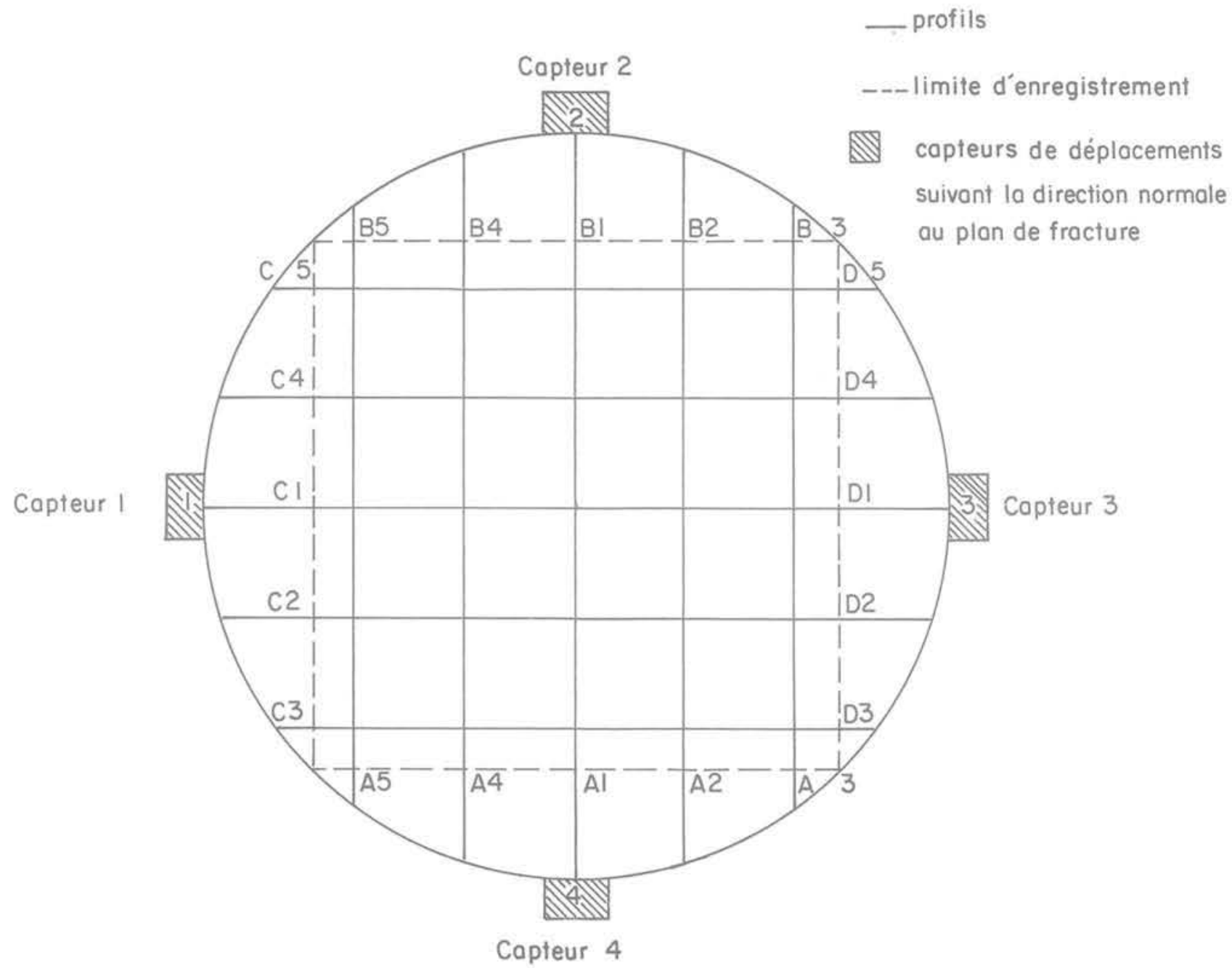

Fig. 5. - Disposition des profils et des capteurs dans le plan de la fracture. 

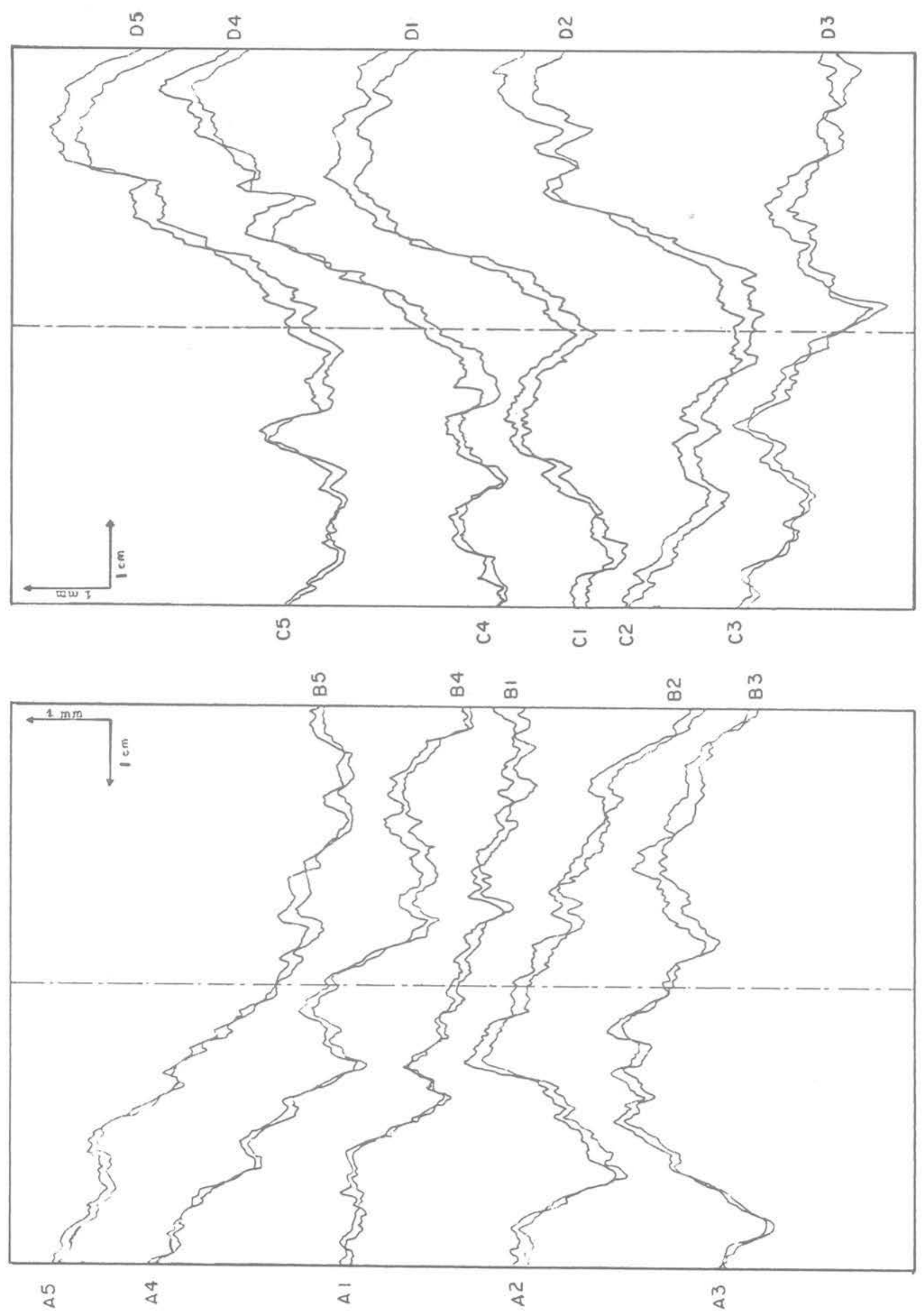

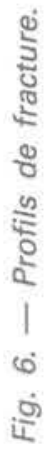




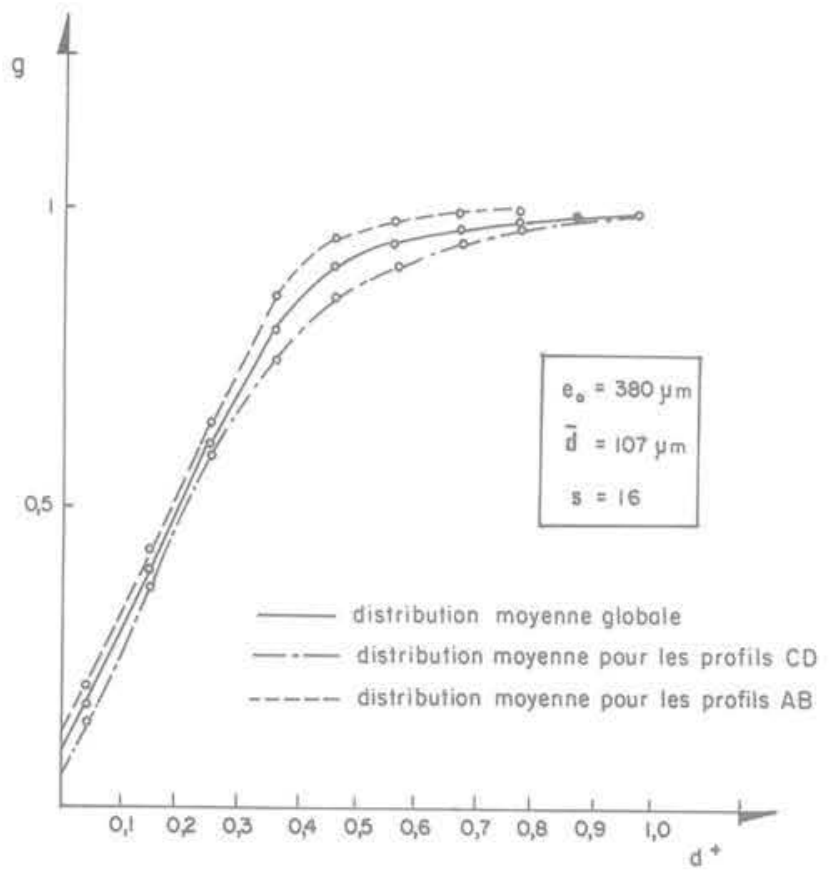

Fig. 7. - Distribution des hauteurs de vide $g\left(d^{+}\right)$.

les profils $C D$ que pour les profils $A B$. La distribution moyenne globale correspond à une fracture comportant de nombreuses petites hauteurs de vide puisque $50 \%$ des hauteurs de vide sont inférieures à $80 \mu \mathrm{m}$ pour une hauteur moyenne de $107 \mu \mathrm{m}$ et une hauteur maximale $\left(e_{0}\right)$ de $380 \mu \mathrm{m}$.

De la courbe $\mathrm{g}\left(\mathrm{d}^{+}\right)$on déduit la loi de variation du degré d'ouverture $\tau\left(e^{+}\right)$(fig. 8) par la relation:

$$
\tau\left(e^{+}\right)=\frac{1-g\left(1-e^{+}\right)}{1-g(0)}
$$

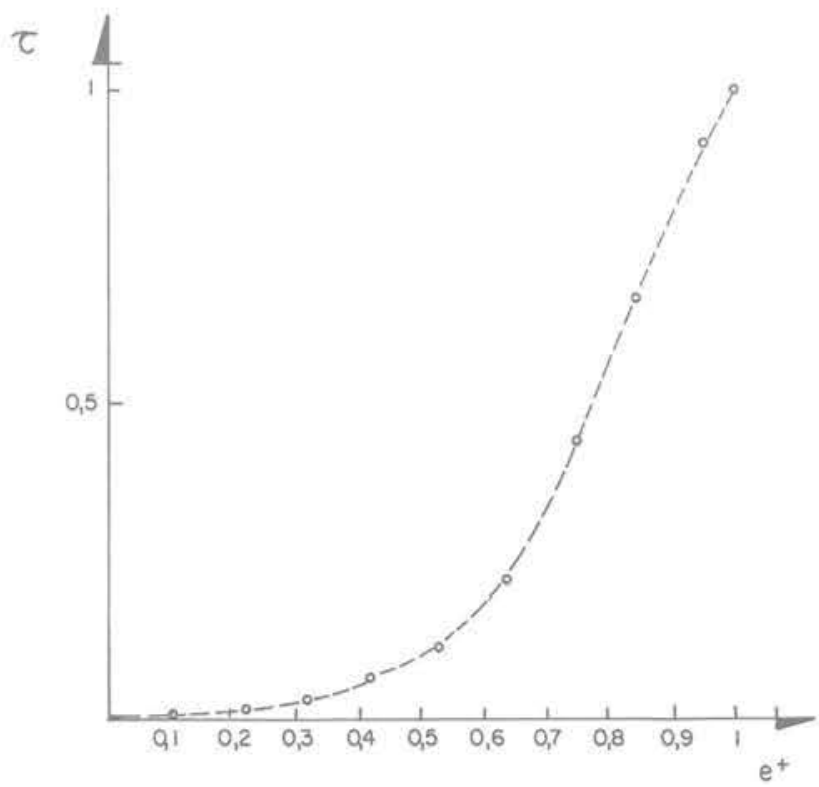

Fig. 8. - Loi de variation du degré d'ouverture en fonction de l'écartement.
Le terme correcteur $1-\mathrm{g}(0)$ prend en compte la méthode de calage des profils.

\subsection{Etude du comportement de la fracture en compression simple}

L'éprouvette est équipée de quatre capteurs linéaires situés aux extrémités des deux diamètres d'enregistrement de profils $\left(A_{1} B_{1}\right.$ et $\left.C_{1} D_{1}\right)$ (fig. 5 et 9). Ces capteurs mesurent les déplacements normaux relatifs des deux épontes de la fracture perpendiculairement au plan de celle-ci. Les valeurs de ces déplacements (qui comprennent la diminution de l'écartement de la fracture et la déformation de la partie rocheuse comprise entre les deux points d'appui des capteurs), ainsi que celle de la force, sont recueillies en fonction du temps sur un enregistreur six voies. Simultanément, on enregistre pour deux des capteurs diamétralement opposés la courbe "contrainte normale-déplacement normal s.

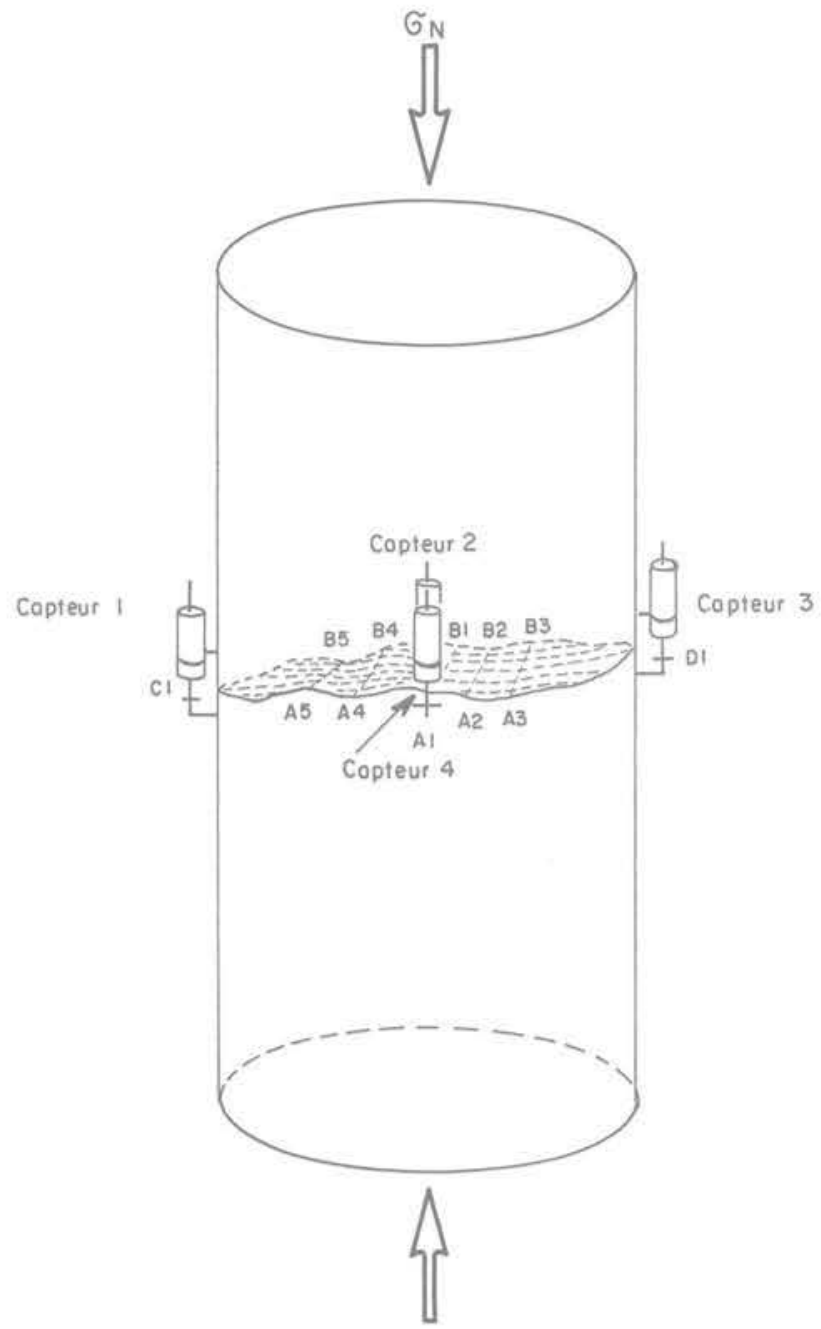

Fig. 9. - Dispositif expérimental pour l'étude du comportement de la fracture en compression simple. 
L'éprouvette est soumise à deux séries de cinq cycles de contraintes croissantes:

$6 \mathrm{MPa}-12,5 \mathrm{MPa}-25 \mathrm{MPa}-50 \mathrm{MPa}-75 \mathrm{MPa}$.

Sur la courbe « contrainte normale-déplacement normal» pour les premiers cycles de chargement (fig. 10),
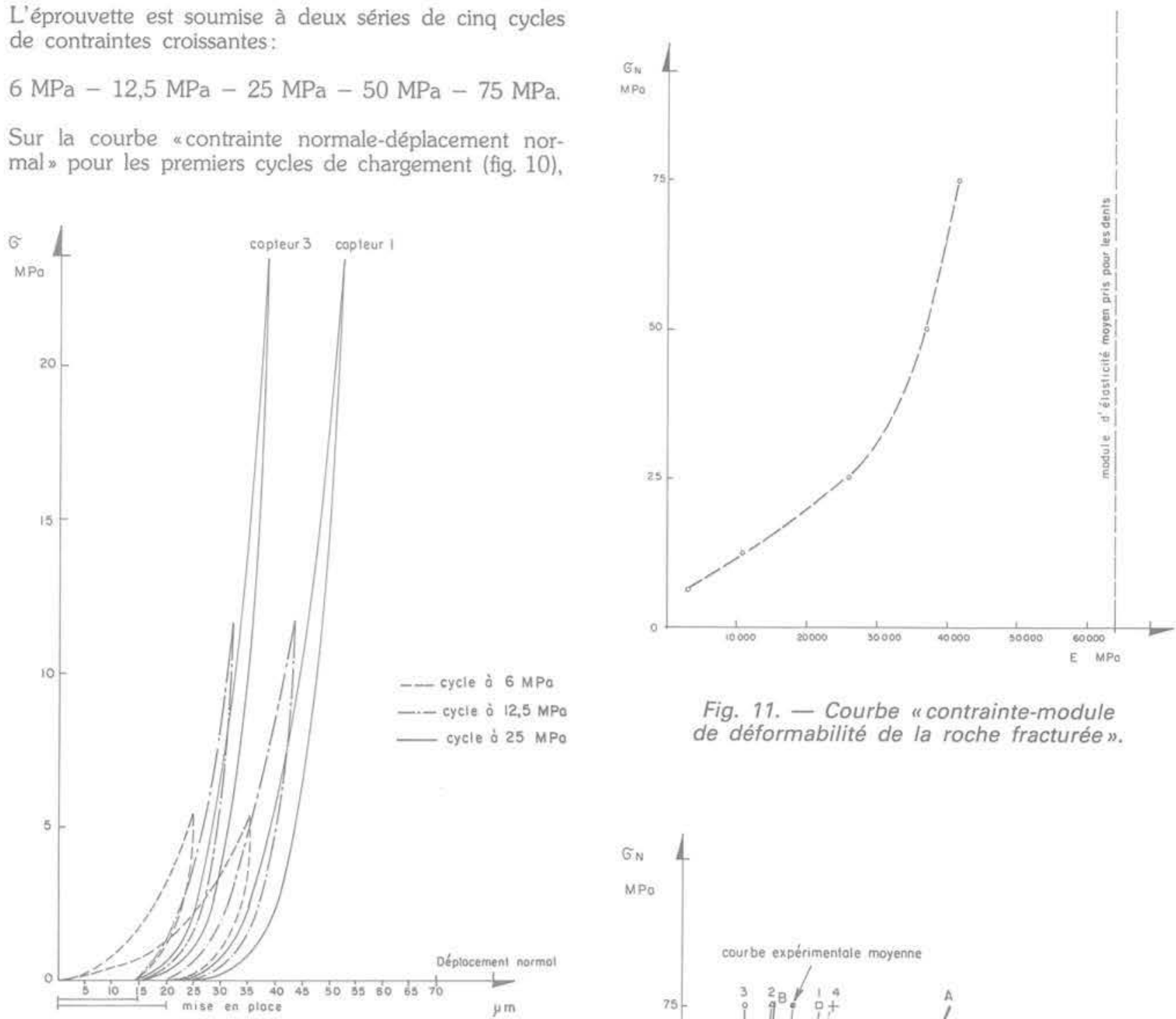

Fig. 11. - Courbe "contrainte-module de déformabilité de la roche fracturée».

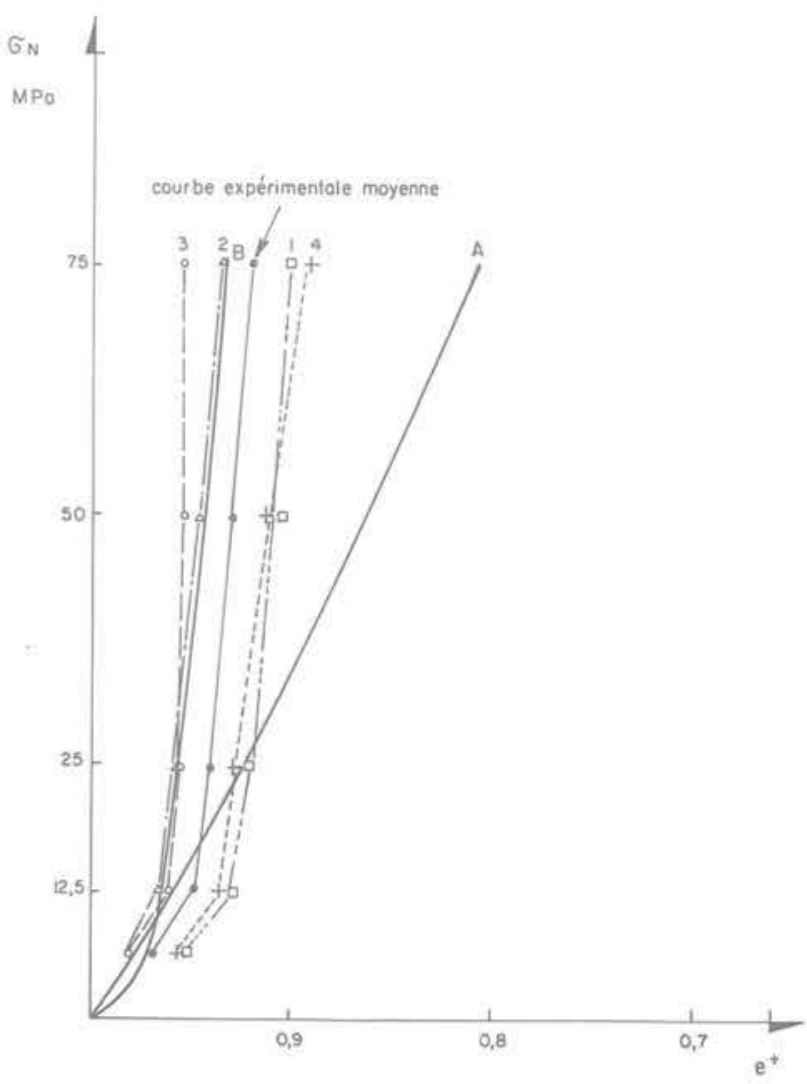

on constate lors du premier cycle à $6 \mathrm{MPa}$ une fermeture irréversible importante qui ne s'accentue pas dans les cycles suivants pour lesquels le comportement est élastique, bien qu'une légère hystérésis subsiste. La « fermeture résiduelle» du premier cycle peut apparaître comme la fin de la mise en place des deux parties de l'éprouvette, une précharge de $0,35 \mathrm{MPa}$, dont l'effet n'est pas représenté sur la figure 10 , ayant déjà provoqué une fermeture moyenne de $28 \mu \mathrm{m}$. D'autre part, lors de la deuxième série de cycles pour cette même contrainte $(6 \mathrm{MPa})$ aucune «fermeture résiduelle» n'est apparue.

En corrigeant la courbe brute de la figure 10 de la * fermeture résiduelle» et du terme dû à l'élasticité de la roche, on trace pour chacun des capteurs la courbe "contrainte normale-écartement" (fig. 11, courbes 1, 2, 3 , 4). On constate que pour tous les capteurs, la diminution de l'écartement reste faible même aux

Fig. 12. - Courbes expérimentales (1, 2, 3, 4) et théoriques $(A, B)$ "contrainte-écartement". 
contraintes les plus élevées puisqu'à $75 \mathrm{MPa}$, l'écartement n'a varié que de 5 à $10 \%$. D'après ces résultats on peut craindre, pour des contraintes plus élevées, d'atteindre le seuil de rupture de l'éprouvette avant d'avoir pu fermer la fracture dans des proportions importantes.

Cette remarque n'est pas contredite par l'évolution du «module du déformabilité " de la roche fracturée évaluée à partir des courbes expérimentales. A la moitié de la résistance à la compression simple de la roche intacte, le "module de déformabilité " de la roche fracturée est déjà égal aux $2 / 3$ du module d'élasticité de la roche intacte (fig. 12).

Lors de la deuxième série de cycles, la variation de l'écartement pour chaque cycle est identique à celle des cucles de la première série de chargements. L'ensemble se comporte de façon parfaitement élastique.

Après le dernier essai, on réenregistre des «profils de rugosité » pour constater un éventuel endommagement de la surface de la fracture. Dans les limites de précision de l'appareil, on ne décèle que quelques rares modifications très localisées de la surface de fracture, qui pourraient être dues aussi bien aux manipulations des éprouvettes qu'aux essais de chargement.

Par un calcul simple, on peut estimer la contrainte normale dans une dent subissant une déformation de $5 \%$, ordre de grandeur des déformations de la fracture sous $75 \mathrm{MPa}$. Cette contrainte normale serait d'environ $3200 \mathrm{MPa}$ soit environ 17 fois la résistance à la compression simple de la roche intacte. Cette observation ainsi que l'absence de modifications nettes des profils de fracture à la suite des essais mentionnés ci-dessus laissent supposer qu'il s'exerce sur chacune des dents en charge une pression de confinement non négligeable sans laquelle elle ne pourrait pas résister.

\section{MODELLES DE COMPORTEMENT MÉCANIQUE D'UNE FRACTURE SOUS CONTRAINTE NORMALE}

\subsection{Premier modèle à «dents non confinées »}

\subsubsection{Principe de base}

Pour chaque valeur de l'écartement $\mathrm{e}^{+}$, on peut définir une hauteur de dent limite $h_{1}$ pour laquelle il $y$ a rupture, toutes les dents de hauteur supérieure à $h_{l}$ étant déjà brisées, et celles de hauteur inférieure étant dans le domaine élastique.

Il vient:

$$
\mathrm{h}_{l}^{+}=\frac{\mathrm{e}^{+}}{1-\frac{\mathrm{q}}{\mathrm{E}}}
$$

avec $\sigma_{\mathrm{L}}$ : contrainte limite de rupture dans une dent $E$ : module d'Young du matériau.

Ceci donne, pour une dent $\propto \mathrm{i} »$, de hauteur $\mathrm{h}^{+}$sous contrainte nulle:

si $h_{i}^{+} \leqslant h_{1}^{+}, \quad \sigma_{i}=\frac{E\left(h_{i}^{+}-e^{+}\right)}{h_{1}^{+}}$(domaine élastique linéaire)

et si $h_{1}^{+}>h_{1}^{+}, \quad \sigma_{\mathrm{i}}=\sigma_{\mathrm{R}}\left(e^{+}\right)$(comportement post-rupture)

avec $\sigma_{1}$ : contrainte normale dans la dent «i»

$\sigma_{\mathrm{R}}\left(e^{+}\right)$: contrainte résiduelle dans toutes les dents rompues, supposée ne dépendre que de l'écartement.

En écrivant l'équilibre d'une surface unité de la fracture sous laction d'une part de la contrainte normale transmise par les épontes, et d'autre part des contraintes normales s'exerçant dans les dents de hauteur initiale supérieure ou égale à $\mathrm{e}^{+}$, il vient:

$$
\underbrace{\sigma_{\mathrm{N}}=\sigma_{\mathrm{R}}\left(\mathrm{e}^{+}\right) \cdot\left(1-\tau\left(\mathrm{h}_{\lambda}^{+}\right)\right.}_{\begin{array}{c}
\text { dents en } \\
\text { post-rupture }
\end{array}}+\underbrace{\int_{\text {domaine élastique }}^{\mathrm{h}_{\prime}^{+}} \mathrm{E} \frac{\left(\mathrm{h}^{+}-\mathrm{e}^{+}\right)}{\mathrm{h}^{+}} \mathrm{T}^{\prime}\left(\mathrm{h}^{+}\right) \mathrm{dh} \mathrm{h}^{+}}_{\begin{array}{c}
\text { dents en } \\
\mathrm{e}^{+}
\end{array}}
$$

En effet,

$1-\mathrm{t}\left(\mathrm{h}^{+}\right)$représente la fraction de surface sur laquelle les dents ont une hauteur supérieure à $\mathrm{h}_{l}^{+}$

$\mathrm{T}^{\prime}\left(\mathrm{h}^{+}\right) \mathrm{dh}^{+}$représente la fraction de surface sur laquelle les dents ont une hauteur comprise entre $\mathrm{h}^{+}$et $\mathrm{h}^{+}+$ $\mathrm{dh}^{+}$.

\subsubsection{Paramètres utilisés et résultats}

La contrainte limite est ici prise égale à la résistance du matériau en compression simple $\sigma_{c}$, ce qui revient bien à considérer les dents comme non confinées lors de leur mise en charge.

Pour tester ce modèle, il faut connaître les deux fonctions $\tau\left(e^{+}\right)$et $\sigma_{R}\left(e^{+}\right)$. On a vu que $\tau\left(e^{+}\right)$est tirée

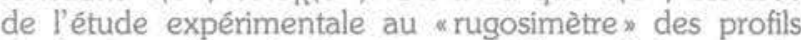
de fracture. Pour la fonction $\sigma_{R}\left(e^{+}\right)$, en l'absence de données expérimentales, on a testé successivement les formules suivantes:

$\sigma_{R}\left(e^{+}\right)=\sigma_{L}$

$\sigma_{R}\left(e^{+}\right)=\left(1-e^{+}\right) \sigma_{L}$

$\sigma_{R}\left(e^{+}\right)=\left(1-2^{2} \sqrt{e+}\right) \sigma_{L}$

$\sigma_{R}\left(e^{+}\right)=\left(2-2^{e^{+}}\right) \sigma_{L}$

La formule retenue a été finalement la plus simple: $\sigma_{\mathrm{R}}\left(e^{+}\right)=\sigma_{\mathrm{L}}$, valeur qui permet de redresser au maximum la courbe "contrainte normale-écartement " obtenue, pour la rapprocher des courbes expérimentales, plus raides que les courbes obtenues avec le 
modèle. Le résultat est représenté par la courbe $A$ de la figure 11. Cette courbe ne correspond pas dans sa forme générale aux courbes expérimentales. D'autre part, avec ce modèle, dès le premier chargement, pour une contrainte ne dépassant pas $1 \mathrm{MPa}$, il existe déjà des dents ayant atteint le seuil de rupture, ce qu'on n'a pas constaté expérimentalement.

Ces observations ont conduit à modifier le modèle initial.

\subsection{Deuxième modèle «à dents confinées"}

\subsubsection{Mise en équations}

On suppose maintenant que la contrainte limite $\sigma_{1}$ varie en fonction de la contrainte de confinement à laquelle est soumise chaque dent en charge. En effet, on considère dans le modèle de fracture, que la différence de hauteur entre deux dents contiguës est faible (fig. 13). Par conséquent, une dent chargée est
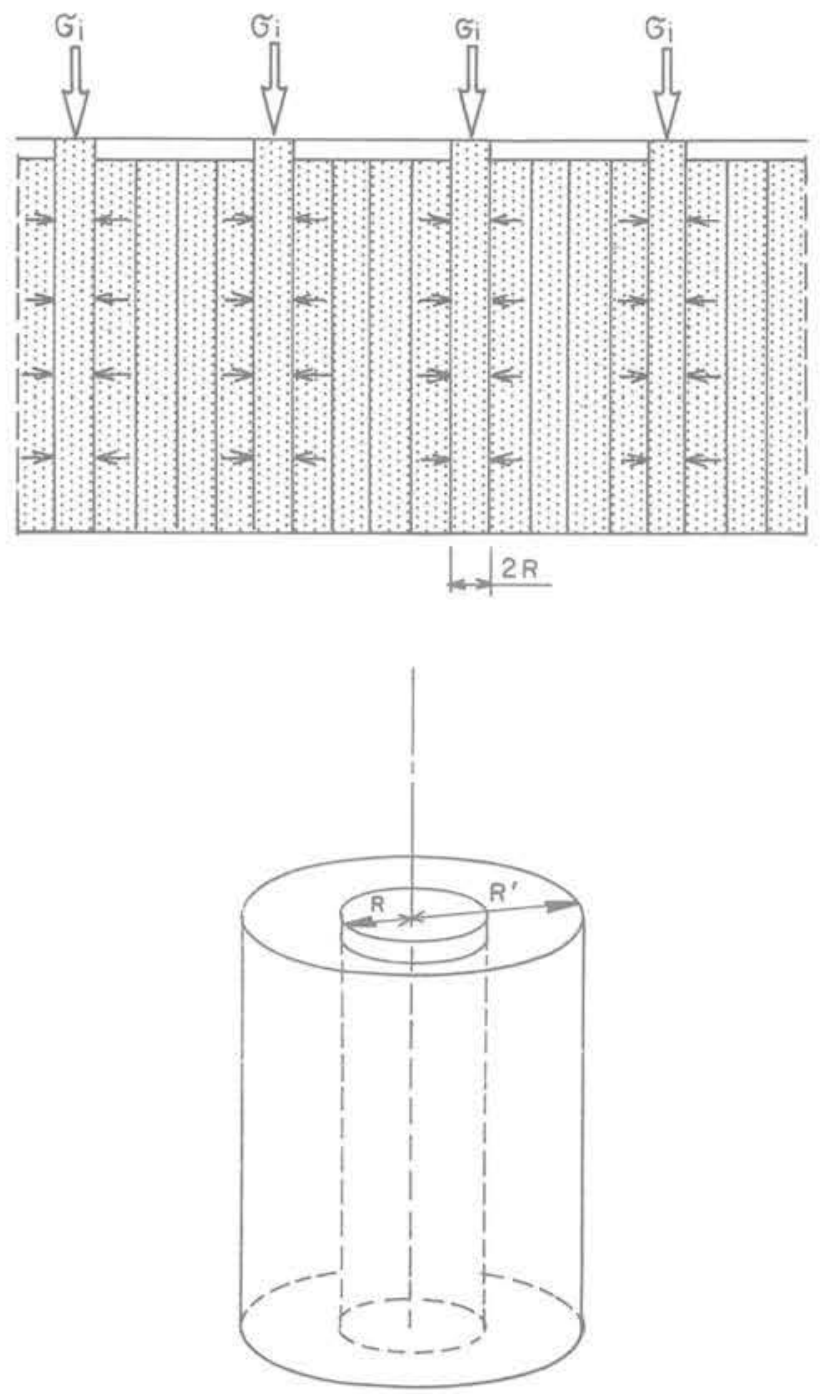

Fig. 13. - Fracture idéalisée (modèle à "dents confinées"). toujours entourée par des dents non chargées qui exercent sur elle une pression de confinement $\sigma_{3 i}$ sur la plus grande partie de sa hauteur. Le rapport de la surface de la zone chargée à la surface totale est donné par: $1-\tau\left(e^{+}\right)$. $\mathrm{Si}$ on modélise une dent chargée et le matériau qui la confine en supposant une symétrie axiale de l'ensemble (fig. 13), le rapport entre les rayons intérieur $\mathrm{R}$ et extérieur $\mathrm{R}^{\prime}$ de l'anneau non chargé est égal à:

$$
\frac{\mathrm{R}}{\mathrm{R}^{\prime}}=\sqrt{1-\mathrm{T}\left(\mathrm{e}^{+}\right)}
$$

En posant comme hypothèse un déplacement radial nul du fait de la présence du matériau environnant, et une contrainte $\mathrm{T} \theta \mathrm{z}$ nulle, la pression de confinement exercée sur chaque dent chargée est:

$\sigma_{3 i}=\frac{v}{1-v} \cdot \frac{R}{R^{\prime}}, \sigma_{i}=\frac{v}{1-v} \cdot \sqrt{1-\tau\left(e^{+}\right)}, \sigma_{i}$

Le critère de rupture choisi est celui défini empiriquement par Brown et Hoek:

$$
\frac{\sigma_{1}}{\sigma_{c}} \geqslant \frac{\sigma_{3}}{\sigma_{c}}+\sqrt{m \frac{\sigma_{3}}{\sigma_{c}}+s}
$$

où $\mathrm{m}$ et $\mathrm{s}$ sont des constantes caractéristiques du matériau, $\sigma_{\mathrm{c}}$ la résistance à la compression simple, $\sigma_{1}$ et $\sigma_{3}$ les contraintes principales.

En combinant les équations précédentes, on obtient une équation du second degré en $\sigma_{\mathrm{L}}$ :

$$
\sigma_{L}^{2} \cdot(1-\beta)^{2}-m \cdot \beta \cdot \sigma_{c} \cdot \sigma_{L}-\sigma_{c}^{2} s=0
$$

avec:

$\beta=\frac{v}{1-v} \cdot \sqrt{1-T\left(e^{+}\right)}$

dont le discriminant est:

$$
\Delta=\left(m^{2} \cdot \beta^{2}+4 \cdot(1-\beta)^{2} \cdot s\right) \cdot \sigma_{c}^{2}
$$

La résolution de cette équation donne la relation suivante:

$$
\sigma_{L}=\sigma_{c} \cdot \frac{\left(m \cdot \beta+\sqrt{\left.m^{2} \beta^{2}+4(1-\beta)^{2} s\right)}\right.}{2(1-\beta)^{2}}
$$

Les valeurs de $\mathrm{m}$ et $\mathrm{s}$ ont été prises respectivement égales à 36 et 1 , valeurs retenues pour un granite écossais par Mc Ewen et al., 1980*.

A partir de l'expression de $\sigma_{\mathrm{L}}$, on peut réécrire les équations du paragraphe 4.1.1., en tenant compte du fait que la relation pour un écartement donné $e^{+}$entre la hauteur de la dent $\mathrm{i}, \mathrm{h}_{\mathrm{i}}^{+}$et la contrainte $\sigma_{i}$ qu'elle subit devient dans le domaine élastique:

$$
\sigma_{i}=\frac{E\left(h_{i}^{+}-e^{+}\right)}{h_{i}^{+}} \cdot \frac{1}{1-\frac{2 v^{2}}{1-v} \cdot \sqrt{1-\tau\left(e^{+}\right)}}
$$

Il vient:

$$
h_{l}^{+}=\frac{e^{+}}{1-\frac{\sigma_{L}}{E} \alpha}
$$

* Mc Ewen T.J., Horseman S.T., Lai S.F. (1980). Geomechanical properties of rocks from the Altnabreac area ENPU 80-13Rapport de l'Institute of geological sciences, Londres. 
avec $\alpha$, coefficient correcteur du confinement;

$\alpha=1-\frac{2 v^{2}}{1-v} \cdot \sqrt{1-\tau\left(e^{+}\right)}$

Et l'équation d'équilibre devient donc:

$$
\sigma_{N}=\sigma_{R}\left(e^{+}\right) \cdot(1-\tau(h+1)
$$

$$
+\frac{1}{1-\frac{2 v^{2}}{(1-v)} \cdot \sqrt{\left.1-\tau^{e+}\right)}}
$$

$$
\int_{e^{+}}^{h_{l}^{+}} E \frac{\left(h^{+}-e^{+}\right)}{h^{+}} \cdot \tau^{\prime}\left(h^{+}\right) d h^{+}
$$

\subsubsection{Résulats obtenus}

Les données introduites sont les mêmes que pour la première version du modèle:

$\left(\tau\left(e^{+}\right), \sigma_{R}\left(e^{+}\right)=\sigma_{L}\left(e^{+}\right)\right)$

Le résultat des calculs correspond à la courbe de la figure 8 , beaucoup plus proche des courbes expérimentales que la courbe $A$ obtenue avec le premier modèle.

\section{CONCLUSION}

Le modèle de base envisagé pour simuler le comportement en compression de fractures dans le granite, suppose la connaissance des variations du degré d'ouverture avec l'écartement, et des contraintes limites et résiduelles dans les dents.

Le rugosimètre utilisé permet d'appréhender le premier de ces paramètres de manière satisfaisante. La prise en compte d'un confinement des dents pour le calcul de la contrainte limite, permet de mieux approcher les résultats expérimentaux.

Les efforts sont maintenant engagés vers une meilleure connaissance des contraintes limites et résiduelles, à la fois par la théorie et l'expérimentation.

\section{Notations}

\section{Morphologie de la fracture}

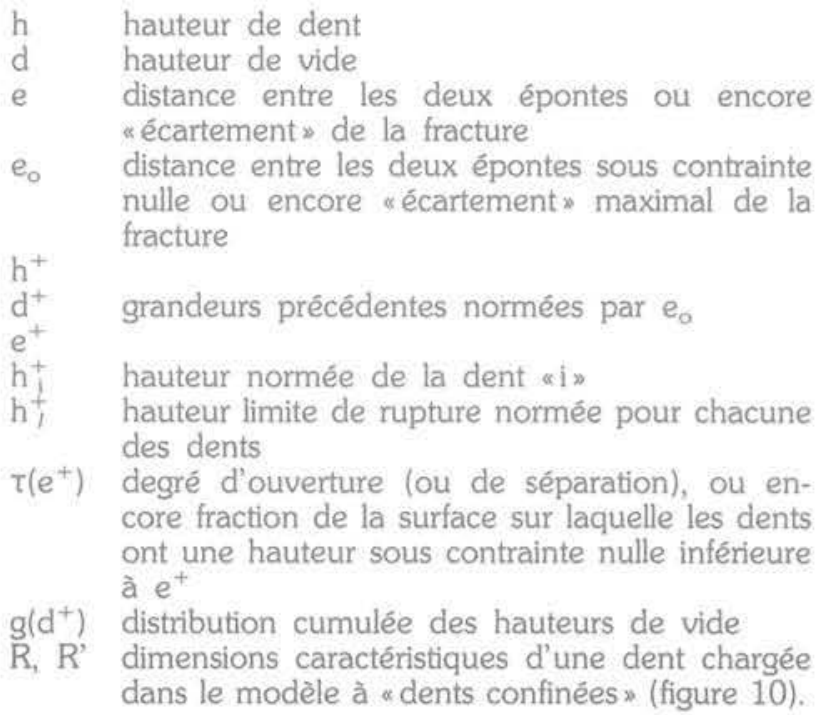

\section{Caractéristiques du matériau}

E module d'Young

$v \quad$ coefficient de Poisson

$\sigma_{c} \quad$ résistance à la compression simple

$s$ écart-type d'une des trois grandeurs précédentes

$\mathrm{s}, \mathrm{m}$ paramètres d'ajustement du critère de rupture pour un matériau donné

\section{Contraintes}

$\sigma_{N} \quad$ contrainte normale appliquée aux ěpontes

$\sigma_{\mathrm{L}} \quad$ contrainte limite de rupture dans une dent

$\sigma_{i} \quad$ contrainte normale dans la dent «i

$\sigma_{31} \quad$ pression de confinement exercée sur une dent chargée par les dents voisines non chargées (modèle à «dents confinées»)

$\sigma_{R}\left(e^{+}\right)$contrainte résiduelle dans toutes les dents rompues, dépendant de l'écartement 
